\title{
Bilateral diffuse uveal melanocytic proliferation secondary to acute lymphoblastic leukemia: a case report
}

\author{
Muhanad Alshathly', Mayada Abu Shanap', Yacoub A. Yousef', Mario Damiano Toro ${ }^{3}$, Reham Shehada', \\ Robert Rejdak ${ }^{3}$, Katarzyna Nowomiejska ${ }^{3}$, Iyad Sultan', Mona Mohammad ${ }^{2}$ \\ 'Department of Pediatric oncology, King Hussein Cancer Center (KHCC), Amman, Jordan \\ ${ }^{2}$ Department of Surgery/Ophthalmology, King Hussein Cancer Center (KHCC), Amman, Jordan \\ ${ }^{3}$ Department of General Ophthalmology, Medical University of Lublin, Poland
}

\begin{abstract}
BACKGROUND: Bilateral diffuse uveal melanocytic proliferation (BDUMP) is a very rare paraneoplastic syndrome that might present with carcinoma. We present a case of an atypical BDUMP secondary to acute B-cell lymphoblastic leukemia (ALL) and report management outcomes at six months follow-up.

CASE PRESENTATION: A 14-year-old female patient with known B-cell ALL presented with blurred vision and headache. Ophthalmological examination revealed new-onset, multiple, round, and pigmented choroidal lesions. Based on the clinical presentation, the diagnosis of BDUMP was suspected. Screening for second malignancies was negative. After nine sessions of plasmapheresis, ophthalmologic examination showed stable, non-progressive retinal lesions with maintained vision and absence of retinal detachment or cataract.

CONCLUSION: This was a rare case of BDUMP in a young patient with a known case of ALL.
\end{abstract}

KEY WORDS: acute lymphoblastic leukemia; bilateral diffuse uveal melanocytic proliferation; retina

Ophthalmol J 2021; Vol. 6, 270-273

\section{INTRODUCTION}

Bilateral diffuse uveal melanocytic proliferation (BDUMP) is a rare paraneoplastic syndrome. This occurrence of bilateral diffuse proliferation of melanocytes in the uvea was first described by Machemer in 1966 [1]. Later in 1982, Barr et al. first identified the BDUMP syndrome [2]. This disease entity is rarely reported in the literature. Until 2017 there were only about 60 cases described [3].
There are five main signs of BDUMP:

- patches at the level of the retinal pigmented epithelium (RPE) in the posterior fundus that are numerous, circular or oval, and subtle;

- early hyper-fluorescence in multifocal areas matching to these patches;

- multiple, slightly raised uveal melanocytic tumors, both pigmented and non-pigmented, as well as signs of diffuse uveal tract thickening 
- exudative retinal detachment;

- rapid progression of cataract formation [4].

The uveal melanocytic proliferation is either caused by a substance secreted by the tumor or an antibody activated by it [5].

Urogenital carcinoma is the most common primary malignancy associated with BDUMP in females $(71 \%)$, with ovarian cancer accounting for $26 \%$ of those. Lung carcinoma is the most common cancer in males, accounting for $51 \%$ of all cases [3]. The presence of early hyper-florescence related to many and circular retinal pigment epithelium atrophic regions, resembling a giraffe pattern, is a prominent distinguishing feature found on fluorescein angiography (FA) [6].

During the two decades from 1980 to 2000 , the incidence of BDUMP cases documented in the literature was 22 cases (1.15 per year) (Ryll et al. 1980). In the last 15 years, 33 new cases (2.2 per year) have been reported, and in the last five years, 22 cases (4.4 per year) have been documented [3]. This increase in BDUMP incidence rates could be related to increasing physician awareness of this condition and an increase in the disease's actual incidence.

In this paper, we present a rare case of BDUMP in a young patient with acute B-cell lymphoblastic leukemia (ALL), which is an uncommon presentation in a young patient that has never been reported before.

\section{CASE PRESENTATION}

The patient is a 14-year-old girl diagnosed with pre-B cell acute lymphoblastic leukemia in August 2018. At the time, she started systemic chemotherapy in accordance with the modified St. Jude total VIIX protocol. Unfortunately, after 24-months from initial diagnosis, she developed combined bone marrow and central nervous system relapses. Routine ophthalmic evaluation at the time of relapse was entirely regular. She was re-induced with a 4-drugs protocol (dexamethasone, daunorubicin, PEG- asparaginase, vincristine with weekly triple intrathecal chemotherapy). Bone marrow and cerebrospinal fluid evaluations post-re-induction were consistent with remission by morphology and negative minimal residual disease (MRD). The re-induction of chemotherapy was complicated by sepsis, superior sagittal sinus vein thrombosis, and pancreatitis, which were all treated successfully.

In December 2020, she presented with complaints of blurred vision, for which ophthalmic eval- uation was done. Eye exam showed normal visual acuity in both eyes (6/6) and a regular anterior segment exam. Dilated fundus exam showed bilateral optic discs swelling, presumed to be related to her sagittal sinus thrombosis, which was in an active stage at that time, and new-onset bilateral multiple round-oval melanocytic lesions, almost $0.5 \mathrm{~mm}$ in diameter, scattered all over the retina in both eyes, with flat retina, and no sub-retinal fluid (Fig. 1). Optical coherence tomography (OCT) scan showed these lesions to be in the sub-retinal pigment epithelium (RPE) space with overlying RPE thinning and dry maculae bilaterally (Fig. 2). Fundus-autofluorescence (FAF) photos showed multiple hypo-auto fluorescent spots giving the characteristic Giraffe-like appearance (Fig. 3). Those new-onset sub-retinal infiltrates were consistent with bilateral diffuse uveal melanocytic proliferation (BDUMP).

The patient was treated with oral acetazolamide and anti-coagulation for her sagittal sinus thrombosis. The natural course of BDUMP is characterized by the progression of the lesions and the development of retinal detachment and cataracts, which usually cause irreversible loss of vision. In order to prevent this progression, the patient was started on prednisolone

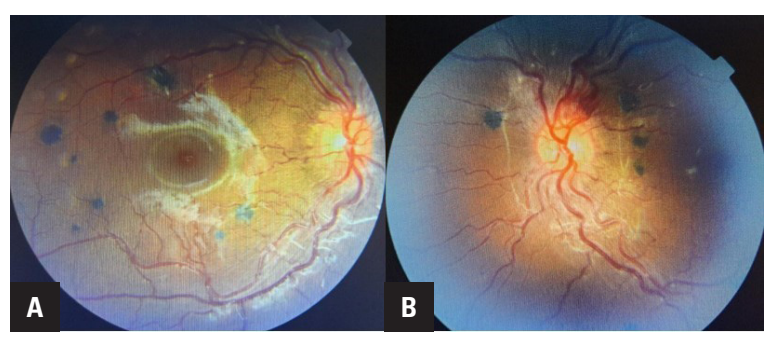

FIGURE 1. Fundus photo for the right eye (A) and left eye (B) at initial eye exam showing bilateral optic disc edema with splinter hemorrhages in (B) and bilateral multiple round-oval melanocytic subretinal infiltrates

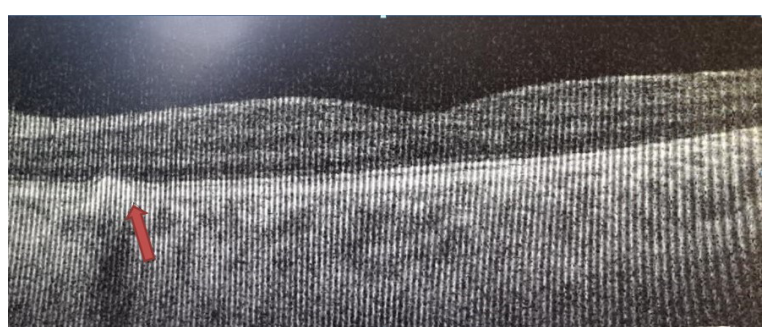

FIGURE 2. Optical coherence tomography (OCT) photo at diagnosis showing dry macula, an area over one of the melanocytic lesions (red arrow) revealed choroidal thickening and shadowing with overlying of RPE-atrophy 


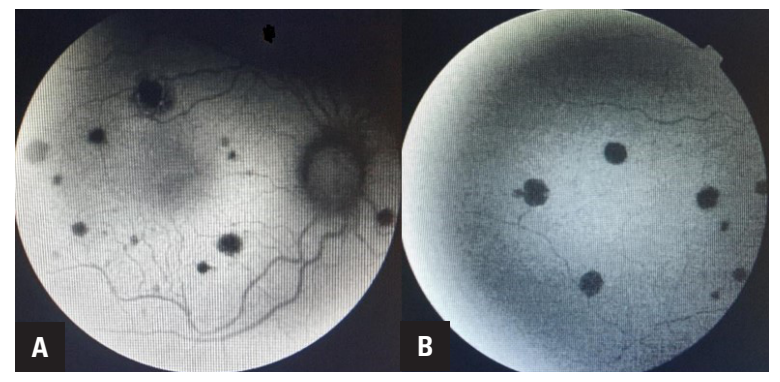

FIGURE 3. Fundus-autofluorescence (FAF) photos for the posterior pole (A) and peripheral retina (B) showing multiple hypo-auto fluorescent spots giving the characteristic "giraffe-like" pattern

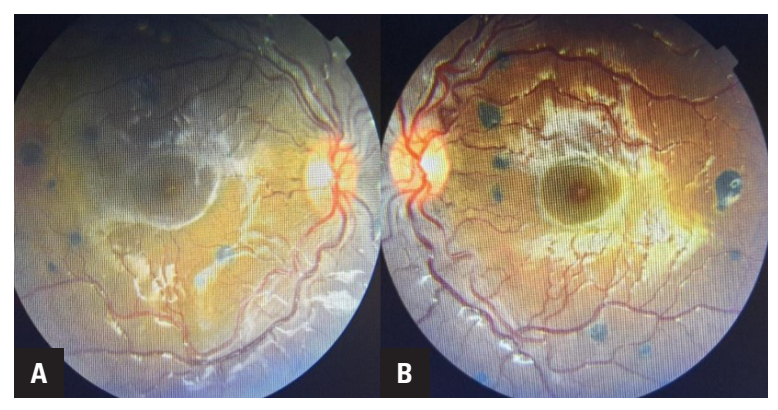

FIGURE 4. Fundus photos for the right eye (A) and left eye (B) after four cycles of plasmapheresis showing resolved optic discs swelling, dry maculae, and stable subretinal infiltrates

$(0.5 \mathrm{mg} / \mathrm{kg} /$ day $)$. After one week, prednisolone was stopped due to worsening the patient's pancreatitis. As an alternative, she was scheduled twice weekly on plasmapheresis. After nine sessions of plasma exchange, her vision remained normal (6/6) bilaterally, the optic discs edema resolved in both sides, and the lesions remained dormant (Fig. 4).

Hereditary cancer panel by next-generation sequencing (NGS) showed a heterozygous MSH6 mutation with: c.2281A\&gt;G (p.Arg761Gly) as a variant of uncertain significance. To exclude occult second cancers as a potential cause of BDUMP, positron emission tomography-computed tomography (PET/CT) was performed and showed no evidence of malignant processes. Upper and lower gastrointestinal tract (GIT) endoscopy was normal. After six months, her ophthalmic condition remained stable. However, the ocular lesion remained unresolved, visual acuity during follow-up 6 months later was $6 / 6$.

\section{DISCUSSION}

This study reported the case of BDUMP syndrome presenting in a 14-year-old patient with ALL and heterozygous MSH6 mutation. To our knowledge, this is the first case study demonstrating such a unique presentation. Even though this is a rare disease, cases of BDUMP in female patients in the literature have been reported in patients with urogenital carcinoma.

The median age at presentation of BDUMP in the literature is 65 years (ranging from 34 to 89 years) [3]. This report is the first to document the case of BDUMP in a 14-year-old child. The chronology between the primary tumor and BDUMP is variable; BDUMP may occur in patients already known to have cancer, as in our patient. BDUMP is also reported to precede the diagnosis of systemic cancer in patients with the bilateral progressive visual deterioration that may start months to years before the diagnosis of systemic cancer [5]

This syndrome has been reported with a wide variety of primary carcinomas. In female patients, urogenital tumors are the most common, while in male patients, lung carcinomas are usually the most common [3]. Of particular interest in our patient is finding MSH6 mutation by hereditary cancer panel, which raised the clinical suspicion of other associated occult malignancies. Therefore, PET-CT scan and upper and lower GIT endoscopy were performed and were all normal. Gass et al. reported bilateral diffuse uveal melanocytic proliferation in patients with occult carcinoma[4]. Rahmy et al. reported bilateral diffuse uveal melanocytic proliferation as a precursor to multiple systemic malignancies such as bladder transitional cell carcinoma and renal clear cell carcinoma [7].

Differentiating BDUMP from the numerous ocular disease entities that mimic it is essential. When this disease was first recognized by Machemer (1960) and later by Barr et al. (1980), the uveal proliferations were thought to result from infiltration of the choroid by malignant ocular melanoma or by choroidal metastases from cutaneous malignant melanoma [1, 2]. Later on, the histopathologic analysis revealed that the infiltration is composed of benign-appearing melanocytes [8].

Patients with BDUMP experience slow, painless, bilateral (usually asymmetric), progressive vision loss over several months. The melanocytic proliferation leads to subretinal infiltration and exudative retinal detachment as a result of outer retinal damage. Visual acuity can also deteriorate due to the development of cataracts, iridocyclitis, or glaucoma [4]. Establishing the diagnosis of BDUMP syndrome does not provide a strong prognostica- 
tion of the length of survival. In virtually all cases, patients died from the dissemination of the primary malignancy [3].

The pathogenesis of BDUMP is not entirely understood. Still, it is thought to involve the production of a growth factor, mitogen, cytokine, or hormone from the systemic carcinoma that leads to the proliferation of melanocytes in the uvea. This hypothesis is supported by in vitro data in which the proliferation of cultured melanocytes was enhanced when treated with the $\operatorname{IgG}$ fraction of human serum from patients with BDUMP [9]. Adamus et al. described two patients who presented with BDUMP in the course of gynecological cancers that had autoantibodies against $35-\mathrm{kDa}, 46-\mathrm{kDa}$, $30-\mathrm{kDa}, 50-\mathrm{kDa}$, and $70-\mathrm{kDa}$ proteins in serum. Genetic analysis of intrabulbar biopsies was reported in two patients. Results showed the cytogenetic finding of a whole gain in chromosome 5 (trisomy 5 ) by single nucleotide polymorphism (SNP) analysis of biopsy material from lesions in each eye in a patient with multiple systemic malignancies [8]. Mittal et al. demonstrated polysomy 8q by multiplex ligation-dependent probe amplification (MLPA) in both eyes in a patient with BDUMP and Endometrial carcinoma [8]. Interestingly, while uveal melanomas commonly involve cytogenetic aberrations in chromosomes 3, 6, and 8 and mutation in genes GNA11, GNAQQ209L, or BRAFV600E; BDUMP syndrome lacks such genetic mutations. These findings support the thesis that BDUMP has a different oncogenic pathway than uveal melanoma [8].

As BDUMP is stimulated by systemic malignan$\mathrm{cy}$, treatment of the primary malignancy and its metastases is very likely to explain some of the reported improvements in visual symptoms and signs. Plasmapheresis is the most successful intervention to improve visual acuity and serous retinal detachment [10]. The mechanism of action for this intervention involves the removal of pathological substances from the blood, such as monoclonal paraproteins and autoantibodies [10]. This patient received nine sessions of plasmapheresis. On follow-up exam, she maintained the normal vision and stable retina lesions in both eyes.

\section{CONCLUSION}

This study presented the unusual case of a young girl presenting with BDUMP secondary to ALL. Genetic testing revealed heterozygous MSH6 mutation. The patient was treated with plasmapheresis. During the six-month follow-up, she maintained good visual acuity and stable retinal lesions.

\section{Conflict of interest}

There are no financial conflicts of interest to disclose.

\section{REFERENCES}

1. Machemer R. [On the pathogenesis of the flat malignant melanoma]. Klin Monbl Augenheilkd. 1966; 148(5): 641-652, indexed in Pubmed: 5996537.

2. Barr CC, Zimmerman LE, Curtin VT, et al. Bilateral diffuse melanocytic uveal tumors associated with systemic malignant neoplasms. A recently recognized syndrome. Arch Ophthalmol. 1982; 100(2): 249-255, doi: 10.1001/archopht.1982.01030030251003, indexed in Pubmed: 7065942.

3. Klemp K, Kiilgaard JF, Heegaard S, et al. Bilateral diffuse uveal melanocytic proliferation: Case report and literature review. Acta Ophthalmol. 2017; 95(5): 439-445, doi: 10.1111/aos.13481, indexed in Pubmed: 28636126.

4. Gass JD, Gieser RG, Wilkinson $C P$, et al. Bilateral diffuse uveal melanocytic proliferation in patients with occult carcinoma. Arch Ophthalmol. 1990; 108(4): 527-533, doi: 10.1001/archopht.1990.01070060075053, indexed in Pubmed: 2322154.

5. O'Neal KD, Butnor KJ, Perkinson KR, et al. Bilateral diffuse uveal melanocytic proliferation associated with pancreatic carcinoma: a case report and literature review of this paraneoplastic syndrome. Surv Ophthalmol. 2003; 48(6): 613-625, doi: 10.1016/j.survophthal.2003.08.005, indexed in Pubmed: 14609707.

6. Chahud F, Young RH, Remulla JF, et al. Bilateral diffuse uveal melanocytic proliferation associated with extraocular cancers: review of a process particularly associated with gynecologic cancers. Am J Surg Pathol. 2001; 25(2): 212-218, doi: 10.1097/00000478-20010200000009, indexed in Pubmed: 11176070.

7. Rahimy E, Coffee RE, McCannel TA. Bilateral diffuse uveal melanocytic proliferation as a precursor to multiple systemic malignancies. Semin Ophthalmol. 2015; 30(3): 206-209, doi: 10.3109/08820538.2013.83 5835, indexed in Pubmed: 24124896.

8. Mittal R, Cherepanoff $S$, Thornton S, et al. Bilateral Diffuse Uveal Melanocytic Proliferation: Molecular Genetic Analysis of a Case and Review of the Literature. Ocul Oncol Pathol. 2015; 2(2): 94-99, doi: 10.1159/000440766, indexed in Pubmed: 27171825.

9. Miles SL, Niles RM, Pittock $S$, et al. A factor found in the $\lg G$ fraction of serum of patients with paraneoplastic bilateral diffuse uveal melanocytic proliferation causes proliferation of cultured human melanocytes. Retina. 2012; 32(9): 1959-1966, doi: 10.1097/ IAE.0b013e3182618bab, indexed in Pubmed: 22791177.

10. Jaben EA, Pulido JS, Pittock $S$, et al. The potential role of plasma exchange as a treatment for bilateral diffuse uveal melanocytic proliferation: a report of two cases. J Clin Apher. 2011; 26(6): 356-361, doi: 10.1002/jca.20310, indexed in Pubmed: 21898575. 